\title{
XXXVII. On the principal screws of inertia of a free or constrained rigid body
}

\author{
Robert S. Ball LL.D. F.R.S.
}

To cite this article: Robert S. Ball LL.D. F.R.S. (1878) XXXVII. On the principal screws of inertia of a free or constrained rigid body, Philosophical Magazine Series 5, 6:37, 274-280, DOI: 10.1080/14786447808639511

To link to this article: http://dx.doi.org/10.1080/14786447808639511

曲 Published online: 13 May 2009.

Submit your article to this journal $\sqsubset \pi$

Џ Article views: 2

Q View related articles $\asymp$ 
Thames $I$ have often observed this. The waves or ripples of the surface cause the sun's rays to enter the water with unequal intensities at different points. At certain points the beams will enter almost without loss by reflexion, and will be traceable by their illuminating the particles of the turbid water. These beams will be nearly parallel to one another; but as they all retreat from the observer, they will appear to converge to a point exactly opposite the sun; to a point within the shadow of his head, in fact, giving the nimbus effect.

The other analogous phenomenon requires to be explained by reflexion. If you stand upon a ridge, or a high wall in the sunlight, so that your shadow falls upon a field of waving corn, you will notice that the corn-field appears to be illuminated in the region all round the shadow of your head. This is best seen when travelling by railway, with the sun about $50^{\circ}$ above the horizon, from the top of an embankment, so that the shadow of the train and observer fall upon the cornfields below.

University College, Bristol. July 31, 1878.

XXXVII. On the principal Screws of Inertia of a Free or Constrained Rigid Body. By ROBERT S. BALL, LL.D., F.R.S., Royal Astronomer of Ireland*.

$\mathrm{T} \mathrm{N}$ the following paper I propose to treat of the effect of an 1 impulse upon a quiescent rigid body, so far as the initial movement of the body is concerned. The analytical investigation of this problem is so well known, that I do not propose to enter into that subject at present. I believe, however, that the ordinary method of viewing the question may be supplemented by the purely geometrical or physical treatment which I shall endeavour to sketch. This geometrical aspect of the problem, as it has presented itself to my mind, has been developed in the course of certain researches in the dynamics of a rigid body which I have ventured to call the Theory of Screws. I shall here indicate the more salient points of this theory which are necessary for the present question.

The most important feature of the geometrical method of viewing the subject is its extreme naturalness, as well as the wide generality with which the problem is grasped. If the rigid body were perfectly free, the questions presented are comparatively simple, so much so that there is not a great deal of interest attached to the investigation. But when we consider the case of a rigid body whose movements are more or

* Communicated by the Author, having been read before the British Association, Dublin, August 1878. 
less constrained, the problems possess a high degree of geometrical interest. It is also the occasion of some surprise that, notwithstanding the infinite variety of conceivable constraints (such as fixed points, axes, contact with fixed surfaces, arrangements of link-work and the like) by which the movements of the rigid body may be hampered, their geometrical classification is of the utmost simplicity. It will be shown that there are six fundamental descriptions of constraint, which include every conceivable arrangement, from leaving the body absolutely free on the one hand, or absolutely immovable on the other. With the investigation of these fundamental forms of constraint we may fitly commence our inquiry.

In the first place, it is to be observed that, as the constraints limit the movements of a body, we may adequately describe the nature of those constraints either by pointing out all the movements of the rigid body which are prohibited, or, on the other hand, by ascertaining. all the movements which are permitted. It is obviously more to the purpose to adopt the latter method of viewing the subject; and therefore we shall proceed to indicate the method by which a complete inventory may be made of the possible movements which a rigid body can execute. It is also to be continually borne in mind that we are only considering the initial movements of the body, and that, consequently, it is only necessary to consider movements which are of indefinitely small magnitude.

Let it therefore be supposed that the rigid body is submitted to our examination when it occupies a definite position $\mathrm{A}$. $\mathrm{We}$ are not now going to apply the impulsive forces to it; we are at present merely making a preliminary trial of a purely geometrical or kinematical character of its capability for displacement. It is at once perceived that the body, not being fixed, can be moved into many closely adjacent positions. Take any one of these positions and call it $B$.

By a celebrated theorem of Chasles it is known that the displacement of the body from $A$ to $B$ can be produced by translating the body parallel to a certain line, and at the same time rotating the body about the same line. We may, for convenience, speak of this motion as a twist; and we may term the angle of rotation the amplitude of the twist. The distance of the translation is proportional to the amplitude of the twist, and may be taken to be equal to the product of the amplitude of the twist and a certain linear magnitude called the pitch. The axis about which the rotation is made, associated with the linear magnitude termed the pitch, constitute what is called a screw. We therefore say that the displacement of the rigid body from $\mathrm{A}$ to $\mathrm{B}$ is effected by a twist about a screw. 
It is to be observed that the twist involves two elemeuts, i.e. a graphic element (the screw), and a metric element (the amplitude of the twist). A little reflection will show that the constraints which permit displacement from $\mathrm{A}$ to $\mathrm{B}$ must also admit of any infinitely small twist being made upon the screw defined by $A$ and $B$. We may therefore say that the body is free to twist about the screw (A, B). If we find on examition that the body cannot be displaced to any position except those which could be attained by twists of suitable amplitude about the screw $(A, B)$, then we can assert that the body has only one degree of freedom; and that freedom is perfectly expressed by the capacity to twist on one definite screw.

It is easy to verify in particular cases the general principle that, no matter what the constraints be, a body which has but one degree of freedom can twist about a certain screw and can have no other movements. For example, a body free to rotate about an axis, but not to slide along it, can only twist about a screw of which the pitch is zero, or a body free to slide along an axis, but not to rotate around it, can only twist about a screw whose pitch is infinite. A less obvious instance is presented in the case of a body of which five points are limited each to a given surface ; but even in this case the body is still only free to twist about a certain screw. Draw the five normals to the surfaces, and regard them as the rays of a linear complex. Then the screw about which the body can twist is the principal axis of that complex, while the pitch of the screw is its parameter. These, however, are only illusstrations; our concern is with the general proportion that when a body has but one degree of freedom, from whatever cause arising, it is free to twist about one screw, and only one.

Suppose, however, it were found that the body, besides being able to twist about a certain screw $a$, was also able to twist about a second screw $\beta$, then twisting about an infinite number of other screws must also be possible. For the position attained by a twist about $a$, followed by a twist about $\beta$, could have been reached by a single twist about some screw $\gamma$. Now as the amplitude of the twists about $a$ and $\beta$ are arbitrary, it is obvious that $\gamma$ must be one of a singly infinite number of screws which include $a$ and $\beta$. It follows that the body must be able to twist about all the screws which constitute the generators of a certain ruled surface.

This surface is called the cylindroid: it is of the third order, its equation being

$$
z\left(x^{2}+y^{2}\right)-2 m x y=0 .
$$

The cylindroid is already well known to the students of the linear geometry of Plïcker. 
A body which has two degrees of freedom is therefore able to twist about all the screws which lie upon a cylindroid. This is true, no matter what be the nature of the constraints. It is a matter worthy of notice that notwithstanding the infinite variety of constraints which would permit a body to have two degrees of freedom, that freedom must still be completely defined by a cylindroid, although all cylindroids are similar surfaces, and possess no variety except as to absolute size. It should also be remarked that the pitches of the screws on the cylindroid are proportional to the inverse squares of the parallel diameters of a certain conic.

If it be found that a body can be twisted about three screws which do not lie upon the same cylindroid, then the body must be capable of being twisted about a doubly infinite number of screws. Of this doubly infinite number three pass through each point in space, all the screws of given pitch lie upon a hyperboloid, and all the screws parallel to a plane lie upon a cylindroid. The pitch of each screw of the system is proportional to the inverse square of the parallel diameter of a certain quadric called the pitch-quadric; and the pitch-quadric is itself the locus of the screws of zero pitch. The entire system is determined when the pitch-quadric is known. These kinematical theorems are intimately connected with Plücker's geometrical speculations on a system of three linear complexes.

Included in the case of freedom of the third order, we have the celebrated problem where a body is rotating around a point. In this case, however, the pitch-quadric assumes an evanescent form, and the general conception of the capabilities of a body which has freedom of the third order are very much degraded.

If a body be able to twist about four screws which do not all belong to such a system as that we have just been describing, then the body must be able to twist about a trebly infinite number of screws. A cone of screws of this system passes through each point of space; and the cone may be drawn by letting fall perpendiculars from the point upon the generators of a cylindroid. It is remarkable that these cones are of the second order; and it can also be shown that the feet of their. perpendiculars upon the generators of the cylindroid are in the same plane. It is thus to be observed that the capabilities of motion possessed by a body which has freedom of the fourth order are completely determined when a certain cylindroid is given in size and position; for then all the cones are determined.

In the case where a body can. twist about five screws not belonging to a system such as that we have just been 
describing, then the system of screws is quadruply infinite; in fact, the body can then twist about one screw of a certain pitch on every line in space.

Finally, if the body can twist about six screws not belonging to the system just mentioned, then the body has freedom of the sixth order, and is, in fact, perfectly free.

We thus see that, corresponding to each order of freedom, a certain group of screws is appropriate ; and we may call such a group a screw-system for the sake of brevity. Thus, in the case of freedom of the second order the screw-system is a cylindroid; in the case of freedom of the fourth order the screwsystem consists of all the screws of proper pitch which intersect a generator of a cylindroid at right angles, and so on.

By this preliminary investigation we are enabled to dismiss entirely all further mention of the constraints. Every conceivable form of constraints can only give the body permission to twist about one of the six types of screw-system. I have not in this brief summary attempted to give any demonstrations of the different theorems involved. For these, reference may be made to the "Theory of Screws'".

We have now laid the foundation of the first part of the problem to be discussed in this paper, inasmuch as we have shown how the body may move; the next question is to ascertain how the body will move when it receives an impulse.

It will, however, be first necessary to consider the most general form of impulse which the body can receive. Now it is well known that all the forces acting upon a rigid body may be reduced to a single force, and a couple in a plane perpendicular to that force. The efficiency of the couple is expressed by its moment; and the moment is the product of a force and a linear magnitude. It will not be unnatural to associate this force and couple with the conception of the screw, already introduced. We may use the expression wrench to denote a force along a screw and a couple in a plane perpendicular to the screw, the moment of the couple being equal to the product of the force and the pitch of the screw. Thus, every system of force acting upon a rigid body constitutes a wrench upon a screw; and it is completely determined when the screw on the one hand, and the force on the other,are both given.

The analogy subsisting between the twist and the wrench, as implied by their mutual connexion with the abstract geometrical conception of a screw, will be the main source of the theorems now to be enunciated.

The original problem has now been brought into this condition. On the one hand, we have a body whose freedom is

* Ball's 'Theory of Screws:' Dublin, 1876. 


\section{of a Free or Constrained Rigid Body.}

expressed by a certain screw-system; and, on the other hand, we have a group of impulsive forces which constitute a wrench on what may be called the impulsive screw. Now, in consequence of this impulse the body will commence to move; but the only motion it can execute must be to twist about some screw of its screw-system; there must therefore be in the screw-system a certain instantaneous screw corresponding to each impulsive screw. If the impulsive screw be given, the instantaneous screw is determined; but the converse is not true. In fact, the instantaneous screw is limited to the screw-system, whereas the impulsive screw may be anywhere in the universe and with any pitch. It is, however, possible to clear away this ambiguity in a very satisfactory manner. It can be shown that, whenever the body is not quite free, there are a group of impulsive screws corresponding to each instantaneous screw. It is not here necessary for us to enter into the properties of these groups of impulsive screws, further than to remark that, if a body receive an impulsive wrench upon any one of the screws belonging to this group, it will commence to twist about the instantaneous screw to which the group is correlated. In each group of impulsive screws there is one screw which merits most particular attention. It can be proved that in each of these groups there is always one serew (but only one) which not only belongs to the group but also belongs to the screw-system expressing the freedom of the body. It therefore follows that the effect of an impulsive wrench anywhere in space could have been produced by an impulsive wrench on a screw suitably chosen from among the members of the screw-system itself. We need therefore only consider the effect of impulsive wrenches upon screws which actually belong to the screw-system.

In this way the ambiguity has been dissipated without any sacrifice of generality : to each screw of the system regarded as an instantaneous screw corresponds another screw of the complex regarded as an impulsive screw, and vice versâ.

The study of this system of correspondence between the impulsive screw and the instantaneous screw of the same screwcomplex, leads to many results of considerable interest, not only on account of their great generality, but also on account of their geometrical character. It is natural to consider, in the first place, whether there are any common elements in the two corresponding systems; and we are thus led to the discovery that when a rigid body has freedom of the $\mathrm{n}$ th order, then $\mathrm{n}$ screws can be selected from the screw-system expressing that freedom such that, if the body receive an impulsive wrench on any one of these scrcus, the body will commence to twist about the same screu. These are called the principal screus of inertia. 


\section{On the Inertia of a Free or Constrained Rigid Body.}

We shall illustrate the existence of these principal screws of inertia by pointing them out in the particular cases of freedom of the second and third orders. When the body has freedom of the second order, the screw-system is a cylindroid. All the generators of a cylindroid are parallel to a plane; and by the anharmonic ratio of four generators is to be understood the anharmonic ratio of four parallel rays drawn through any point. Now it can be shown that the anharmonic ratio of four instantaneous screws on the cylindroid is equal to the anharmonic ratio of the four corresponding impulsive screws. When, therefore, three impulsive screws and the three corresponding instantaneous screws are known, the instantaneous screw corresponding to any impulsive screw is at once determined by geometry. The double rays of the two equianharmonic systems must of course be parallel to the two principal screws of inertia on the cylindroid; and thus the problem of finding the principal screws of inertia for freedom of the second order is completely solved.

In the case of freedom of the third order, the three principal screws of inertia can. also be completely determined by geometry. For this purpose it is necessary to construct a certain ellipsoid, which is defined by the following theorem.

The kinetic energy of a rigid body when twisting with a given twist velocity about any screw of a screw-system of the third order is proportional to the inverse square of the parallel diameter of a certain ellipsoid.

If this ellipsoid be made concentric with the pitch-quadric, it will be possible to draw a triad of common conjugate diameters to the two surfaces; and the required principal screws of inertia are the three screws of the complex which are parallel to these conjugate diameters.

In that special case of freedom of the third order in which a body is rotating about a fixed point, then the general property of the three principal screws of inertia degrades to the well-known property of the principal axes. It will be observed that the theory here propounded may be considered to generalize the property of the principal axes into a general property for freedom of the third order, and then further into a property for freedom of any order.

$W_{\theta}$ conclude by pointing out the six principal screws of inertia of a perfectly free rigid body. They are found as follows :-Draw the three principal axes, A, B, C, through the centre of gravity, and let $a, b, c$ be the radii of gyration; then two screws on $A$ with the pitches $+a,-a$, and two similar screws on B and on C, constitute the six principal screws of inertia.

Dunsink, Co. Dublin, August 1878. 